\title{
The Genomic Applications in Practice and Prevention Network
}

\author{
Muin J. Khoury, $M D, P h D^{I}$, W. Gregory Feero, $M D, P h D^{2}$, Michele Reyes, $P h D^{I}$, Toby Citrin, $J D^{3}$, \\ Andrew Freedman, PhD ${ }^{4}$, Debra Leonard, $\mathrm{PhD}^{5}$; and the GAPPNet Planning Group: \\ Wylie Burke, MD, PhD ${ }^{6}$, Ralph Coates, PhD ${ }^{1}$, Robert T. Croyle, PhD ${ }^{3}$, Karen Edwards, PhD ${ }^{7}$, \\ Sharon Kardia, $P h D^{2}$, Colleen McBride, $P h D^{2}$, Teri Manolio, $M D, P h D^{2}$, Gurvaneet Randhawa, $M D^{8}$, \\ Rebekah Rasooly, $P h D^{9}$, Jeannette St. Pierre, $M P H^{1}$, and Sharon Terry, $\mathrm{MS}^{10}$
}

\begin{abstract}
The authors describe the rationale and initial development of a new collaborative initiative, the Genomic Applications in Practice and Prevention Network. The network convened by the Centers for Disease Control and Prevention and the National Institutes of Health includes multiple stakeholders from academia, government, health care, public health, industry and consumers. The premise of Genomic Applications in Practice and Prevention Network is that there is an unaddressed chasm between gene discoveries and demonstration of their clinical validity and utility. This chasm is due to the lack of readily accessible information about the utility of most genomic applications and the lack of necessary knowledge by consumers and providers to implement what is known. The mission of Genomic Applications in Practice and Prevention Network is to accelerate and streamline the effective integration of validated genomic knowledge into the practice of medicine and public health, by empowering and sponsoring research, evaluating research findings, and disseminating high quality information on candidate genomic applications in practice and prevention. Genomic Applications in Practice and Prevention Network will develop a process that links ongoing collection of information on candidate genomic applications to four crucial domains: (1) knowledge synthesis and dissemination for new and existing technologies, and the identification of knowledge gaps, (2) a robust evidence-based recommendation development process, (3) translation research to evaluate validity, utility and impact in the real world and how to disseminate and implement recommended genomic applications, and (4) programs to enhance practice, education, and surveillance. Genet Med 2009:11(7):488-494.
\end{abstract}

Key Words: decision support, genomics, information, medicine, network, public health

T he ongoing success of genome wide association studies (GWAS) in uncovering genetic risk factors for many common diseases has fuelled expectations of a new era of health care based on personalized treatment, early detection, and disease prevention. ${ }^{1-3}$ An optimal process is needed for appropriate translation of these new genomic discoveries into practice. The process should include mechanisms for developing an understanding of the relationship between these newly discov-

From the ${ }^{1}$ Office of Public Health Genomics, Centers for Disease Control and Prevention, Atlanta, Georgia; ${ }^{2}$ National Human Genome Research Institute, National Institutes of Health, Bethesda, Maryland; ${ }^{3} \mathrm{Center}$ for Community and Public Health Genomics, University of Michigan, Ann Arbor, Michigan; ${ }^{4}$ Division of Cancer Control and Population Sciences, National Cancer Institute, National Institutes of Health, Bethesda, Maryland; ${ }^{5}$ Department of Pathology and Laboratory Medicine, Cornell University, New York City, New York; ${ }^{6}$ Center for Genomics and Healthcare Equality, University of Washington, Seattle, Washington; ${ }^{7}$ Center for Genomics and Public Health, University of Washington, Seattle, Washington; ${ }^{8}$ Agency for Healthcare Research Quality, Rockville, Maryland; ${ }^{9}$ National Institute for Diabetes, Digestive and Kidney Diseases, National Institutes of Health, Bethesda, Maryland; and ${ }^{10}$ Genetic Alliance, Washington, DC. ered factors and clinical outcomes (clinical validity), and the costs, benefits, and harms of genome-based technologies in real world settings (clinical utility). ${ }^{4}$ Furthermore, the process should facilitate the development of evidence-based guidelines for the use of genomic applications 5 ; and appropriate implementation of these applications in practice, including protection of individuals and communities against discrimination based on genetic information. ${ }^{6}$ Importantly, advances in genomics should be considered in the context of the larger forces affecting health care delivery in the United States, including escalating costs, differential access to quality health care, and a growing number of uninsured persons in our population. The viability of genomics in health and health care will be fundamentally related to its ability to demonstrate clinical utility and cost-effectiveness in an already strained system.

This article discusses the development of an open and collaborative means for both building the evidence base for emerging genomic technologies and transitioning validated technologies into clinical and public health practice. This collaboration complements and builds on the existing Centers for Disease Control (CDC) initiative, the Evaluation of Genomic Applications in Practice and Prevention (EGAPP) (described in more detail later, see Ref. 7) and extends it to involve genomics translation research and programs to accelerate knowledge synthesis and dissemination of available information.

\section{WHY DO WE NEED A NEW COLLABORATIVE INITIATIVE? A HYPOTHETICAL CASE STUDY}

To illustrate how a collaborative mechanism to develop, synthesize, and disseminate credible information could benefit various stakeholders, consider a hypothetical commercial genetic test proposed for use by health care providers - a panel of genetic markers to aid selection of drug choice and dosing for the management of type 2 diabetes. Currently, this type of test would probably emerge from data amassed by investigatordriven GWAS, then be packaged as a test by a laboratory or pharmaceutical company, and marketed to health care providers and consumers. At the time the test enters the market, minimal

Muin J. Khoury, MD, PhD, Office of Public Health Genomics, Centers for Disease Control and Prevention, Atlanta, GA. E-mail: mkhoury@cdc.gov.

The report does not necessarily reflect the views and policies of the Department of Health and Human Services.

Disclosure: The authors declare no conflict of interest

Submitted for publication October 31, 2008.

Accepted for publication March 3, 2009.

Published online ahead of print June 3, 2009.

DOI: 10.1097/GIM.0b013e3181a551cc 
Table 1. The Genomic Applications in Practice and Prevention Network (GAPPNet)

\begin{tabular}{ll}
\hline Question & Description and examples \\
\hline What is it? & $\begin{array}{c}\text { Collaboration of individuals and organizations interested in translating appropriately validated } \\
\text { genomic applications into practice and prevention }\end{array}$ \\
What is the vision? & $\begin{array}{c}\text { To realize the promise of genomics in treating and preventing disease, improving health, and } \\
\text { reducing health disparities }\end{array}$ \\
What is the mission? & $\begin{array}{l}\text { To accelerate and streamline the effective integration of validated genomic knowledge into the } \\
\text { practice of medicine and public health in the United States, by empowering research and } \\
\text { evaluation, and disseminating high-quality information on promising genomic applications in } \\
\text { practice and prevention }\end{array}$
\end{tabular}

Who are the members?

Who are the conveners?

What are the domains of GAPPNet?

1. Knowledge synthesis and dissemination

2. Evidence-based guideline development

3. Translation research

4. Translation programs
Initial group of genomics translation grant awardees from NIH, CDC, and other groups; various stakeholder groups; GAPPNet knowledge synthesis developers; other collaborators

$\mathrm{CDC}$ and $\mathrm{NIH}$

Integration of evidence reviews and recommendations into clinical decision support tools via the development of GAPPNet knowledge database

Evaluation of Genomic Applications in Practice and Prevention (EGAPP) Working Group provides independent assessment, develops guidelines, and identifies knowledge gaps

Evaluating clinical utility of pharmacogenomics (e.g., VKORCl testing as an adjunct to anticoagulation). Evaluating the effectiveness of interventions to increase the implementation of evidence-based tests (e.g., BRCA1)

Promoting development and implementation of model programs (e.g., integrating validated genomic applications in proactive and improving health care quality). Sponsoring educational activities for providers (e.g., appropriate use of BRCA1 testing). Promote the conduct of population health surveillance (e.g., impact of direct to consumer personal genomics) evidence might be available to stakeholders regarding the test's analytic and clinical validity, and it is probable that no clinical utility information would be available. Although the laboratory offering the test may be Clinical Laboratory Improvement Act approved, the test itself may not have undergone Food and Drug Administration (FDA) approval (because this is a laboratory developed test). Unfortunately, the test could reach the market long before research has been conducted to assess the test's clinical utility, and comparative effectiveness vis-à-vis other existing approaches that do not use genetic testing. The hypothetical diabetes test could thus meet with substantial skepticism by researchers, provider groups, insurers, public health institutions, and policymakers. This would limit its potential for reimbursement, uptake, and, ultimately, public health benefit. On the other hand, consumer interest in the test could also create a supply-demand chain that propels the test into clinical use before adequate evidence has been established.

A collaborative model for facilitating and coordinating the translation of genomic applications into health care and population health could dramatically improve the process for all groups. Returning to the example of the hypothetical diabetes test, one can envision several key functions that a coordinated system for translation might provide. First, all stakeholders could benefit from an unbiased initial assessment of the proposed test. Based on preliminary data, the assessment could define analytic validity, clinical validity, and potential clinical utility of the test to various stakeholders, and identify gaps in the knowledge base required for downstream acceptance and/or reimbursement by the public, care providers, and insurers. This type of early premarket assessment would permit stakeholders to develop priorities for funding, and potentially spur private investment in clinical utility studies required for reimbursement by third party payers. Second, all stakeholders could benefit from an enhanced means for communication among and within groups. A means to align priorities and coordinate translation efforts could substantially reduce duplicative spending and time delay, and create an "evidence match" between the evidence generation process and the priorities of insurers, health care providers, and policy makers. Finally, all stakeholders could benefit from a structured means for coordinating evidence synthesis, dissemination, and educational efforts (for both health care providers and the public) in the immediate premarket and postmarket phase of test development and deployment. Currently, even the most promising applications may not reach a wide audience because of a lack of effective migration of evidence into national health care guidelines and the inefficient application and uptake of those guidelines in the health care sector. Coordination of these activities is critical to realizing the benefit of any new technology and has been recognized as a major stumbling point in the translational continuum. ${ }^{8,9}$

\section{THE GENOMIC APPLICATIONS IN PRACTICE AND PREVENTION NETWORK INITIATIVE}

We believe that the development and availability of reliable and updated information on genomic applications in health care practice in the United States can be accelerated by a new collaborative initiative (Table 1). At the heart of the initiative is the convening of stakeholders interested in translating high impact, appropriately validated genomic applications into practice and prevention. Stakeholders key to the success of Genomic Applications in Practice and Prevention Network (GAPPNet) include academia, government, health care and public health 
professionals, behavioral and social scientists, health care payers and plans, policy makers, media, disease-specific organizations, business, the biotechnology and pharmaceutical industries, educators, and information technology developers. The vision of GAPPNet is to realize the promise of genomics (and related fields) in treating and preventing disease, improving health, and reducing health disparities. The mission of GAPPNet is to accelerate and streamline the effective integration of validated genomic knowledge into the practice of medicine and public health in the United States, by empowering and sponsoring research, evaluating research findings, and disseminating high-quality information on candidate genomic applications in practice and prevention. The premise of GAPPNet is that there is a chasm between gene discoveries and their clinical validity and utility for successful applications in health care and disease prevention. This chasm is due to the lack of readily accessible information about the validity and utility of most genomic applications and the lack of necessary knowledge by consumers and providers to implement what is known. Therefore, there is an urgent need to: (1) track information on the clinical validity and utility of genomic applications in health practice; (2) facilitate the uptake and use of objective information from evidence reviews and technology assessments in a variety of health care settings; (3) identify additional knowledge gaps that drive clinical and population research; (4) highlight the need for targeted research on the clinical validity and utility of these candidate applications; (5) support best practices through policy development, education and information dissemination; and (6) highlight the need for postmarket population surveillance and applied research. GAPPNet would not replace, but complement and partner with, the many coordinating efforts in genomics already ongoing in the United States among both professional specialty organizations, advisory groups to the government (e.g., Secretary's Advisory Committee on Genetics, Health and Society) and multigroup entities (partial list in Table 2). GAPPNet will provide a stakeholder forum for these groups toward the goal of genomic integration into health care and disease prevention. In addition, GAPPNet will interface with international collaborations (e.g., with the Genome-based Research and Population Health International Network ${ }^{10}$ ), and international health technology assessment groups. ${ }^{11}$

It is important to acknowledge at the outset that genomics technologies are not different from other health care technologies and they need to follow principles of evidencebased medicine and comparative effectiveness. Therefore, GAPPNet could also leverage several ongoing nongenomic health care federal and nonfederal initiatives to integrate gene-based applications in the context of medicine and population health (e.g., ${ }^{12-18}$ ).

\section{GAPPNet ACTIVITIES}

Details of how GAPPNet will be set up and operated will be discussed over the next few months by the GAPPNet planning group. In brief, GAPPNet will develop a process that links ongoing collection of information on candidate genomic applications to four crucial domains: (1) knowledge synthesis and dissemination for new and existing technologies, and the identification of knowledge gaps, (2) a robust evidence-based recommendation development process (built on the EGAPP initiative, see later), (3) translation research to evaluate validity, utility and impact in the real world and how to disseminate and implement recommended genomic applications, ${ }^{19}$ and (4) programs to enhance quality of care in practice, education, and surveillance. Activities in the four domains currently exist but

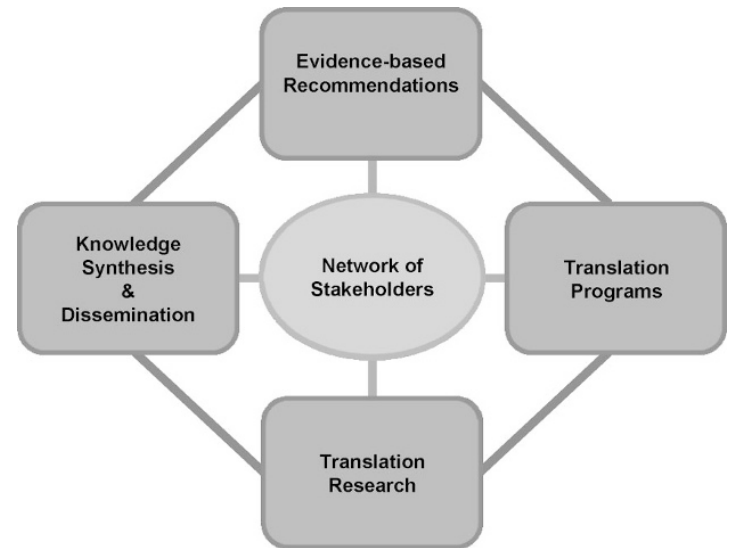

Fig. 1. The Genomic Applications in Practice and Prevention Network (GAPPNet).

will be strengthened and extended with this initiative. Key to the success of GAPPNet is an expanded stakeholder group to ensure collaboration and representation of multiple viewpoints. Although GAPPNet is largely a convening entity, resources to implement activities in the four domains will be developed and identified by the stakeholder group through public and private partnerships. The interrelationship of GAPPNet domains is shown in Figure 1.

\section{GAPPNet Domain 1: Knowledge synthesis and dissemination}

GAPPNet will promote the objective synthesis and timely dissemination of information on candidate health applications of genome-based tests and technologies. A central website (the GAPPNet knowledge base) will maintain and update a list of these applications with links to partner websites and available credible information (e.g., GeneTests ${ }^{20}$ ). GAPPNet will expand the information based on genomic application topics that have been identified and/or reviewed by the EGAPP working group (see later). GAPPNet will sponsor a knowledge synthesis process that uses and adapts methods of horizon scan and rapid systematic reviews developed by EGAPP. This process will use standardized formats to synthesize and update available information on these applications. For each suggested application and health-related scenario, information will be accumulated on analytic validity, clinical validity, clinical, and public health utility using methodologies of systematic reviews and evaluations. When evidence-based reviews have been conducted by EGAPP $^{7}$ or other health technology assessment groups such as the US Preventive Services Task Force ${ }^{21}$ (USPSTF) the website will provide links to these reviews. In the absence of comprehensive reviews, brief reports of selected promising genomic applications, e.g., those identified by EGAPP horizon scans will be provided. When no information is available, this will also be pointed out. The GAPPNet knowledge base thus will provide an initial and credible stop for users to scan for information on available genomic applications and what we know and what we do not know with respect to their analytic and clinical performance. GAPPNet collaborators conducting research and evaluation (see later) will provide important contributions to the GAPPNet knowledge base contents and dissemination. Partnerships will be sought with key medical and public health journals to publish results of knowledge synthesis evaluations similar to the current arrangement the EGAPP Working Group has with 
Genetics in Medicine, a pioneer in the intersection of genomic medicine and evidence-based medicine. A GAPPNet knowledge base working group will be formed to determine the content and curate the site.

A related and crucial function of GAPPNet is the systematic and active dissemination of the knowledge base to consumers, policy makers, and providers. Statements relating the evidence supporting an application (or lack thereof) will be made available on an ongoing basis to inform provider and consumer choices. Further, it is anticipated that these statements will be used to support the development of clinical decision support tools for clinical practice and disease prevention. To this end, GAPPNet will actively engage professional organizations related to the fields of practice (e.g., family practice, oncology, cardiology) and relevant stake holders from health informatics. The EGAPP Stakeholder Group, made up of over 30 professionals, has already made an impressive start on this work (see later) and is expected to play a key leadership role in GAPPNet.

\section{GAPPNet Domain 2: Development of evidence-based recommendations}

EGAPP represents a fundamental building block for GAPPNet. In 2004, CDC launched the EGAPP initiative. ${ }^{7}$ The main goal of EGAPP is to establish and test a systematic, evidence-based process for evaluating genetic tests and other applications of genomic technologies that are in transition from research to clinical and public health practice. EGAPP has integrated existing recommendations on the evaluation of interventions from professional organizations and advisory committees, task forces (e.g., USPSTF, CDCs Task Force on Community Preventive Services), and international health technology assessment groups. EGAPP activities are focused around the independent, nonfederal EGAPP Working Group established in 2005. The roles of this multidisciplinary panel include developing methods and processes for evidence reviews of complex and rapidly emerging technologies, including, identification, prioritization and selection of topics, guidance of the conduct of evidence reviews, and development of recommendations for clinical and public health practitioners based on the evidence. ${ }^{22}$ As of early 2009, Agency for Healthcare Research and Quality (AHRQ) Evidence-based Practice Centers have completed a total of five EGAPP-commissioned evidence reports: (i) genomic tests for ovarian cancer detection and management; (ii) testing for cytochrome P450 polymorphisms in adults with non-psychotic depression treated with selective serotonin reuptake inhibitors (SSRIs); (iii) hereditary nonpolyposis colorectal cancer: diagnostic strategies and their implications; (iv) impact of gene expression profiling tests on breast cancer outcomes. Other contracted groups have completed one additional EGAPP-commissioned evidence report, on UGT1A1 genotyping and morbidity and mortality in patients with metastatic colorectal cancer treated with irinotecan, and a supplemental report, on DNA testing strategies aimed at reducing morbidity and mortality from Lynch syndrome. Based on consideration of the information provided by the evidence report and significant clinical and social contextual issues about a specific genetic test, the EGAPP Working Group develops a recommendation statement that summarizes the current knowledge about the validity and utility of the genetic test, provides guidance on appropriate use of the test, and defines key knowledge gaps and needed research. In 2007, the first in a series of EGAPP Working Group recommendation statements was published on CYP450 testing in patients with depression treated with SSRIs. Three other reports were just published in this journal. ${ }^{23-25}$
An EGAPP Stakeholders Group was set up in 2005 to provide feedback to the Working Group, and to assist with dissemination of the recommendation statements to various organizations. The Stakeholders Group includes representatives from academia, health care and public health professionals, health care payers and plans, policy makers, media, consumer advocacy groups, business, the biotechnology industry, educators, and information technology developers. The broad-based EGAPP Stakeholder Group will be a key component of GAPPNet in supporting the dissemination of evidence-based recommendations and the implementation of those recommendations into practice with their constituent stakeholder groups. Through the other three domains of GAPPNet, there will be an explicit connection between EGAPP recommendations and the implementation of translation programs and research, as well as dissemination of information coming out of EGAPP and other evidence-based groups. We envision that the GAPPNet stakeholder group will build on the current EGAPP Stakeholder Group to include representatives from other initiatives (e.g., Table 2). The GAPPNet Stakeholder Group will support dissemination of credible information on genomic applications for which evidence is lacking about their validity and/or utility.

\section{GAPPNet Domain 3: Translation research}

An important feature of GAPPNet is the promotion of translation research in multiple disciplines needed to close the knowledge gaps about candidate genomic applications in practice. Basic and translation research could be sponsored by federal agencies, the private sector, or public-private partnerships. A substantial start on both gene discovery and early translation research regarding issues in genetic epidemiology surrounding GWAS has been made by the Office of Population Genomics of the National Human Genome Research Institute (NHGRI) (http://www.genome.gov/19518660). Addition of GWA genotyping to existing case-control studies, cohort studies, clinical trials, and biorepositories, and cataloging of results of GWAS (http://www.genome.gov/26525384) will help to develop and synthesize genomic knowledge for clinical applications. Despite these efforts, the EGAPP initiative has uncovered major gaps in our knowledge base on the clinical validity and utility of even the most promising genomic applications in practice and prevention. Between 2001 and 2006, the USPSTF recommended only one genetic test for use in primary care, largely because of a lack of a sufficient evidence base. ${ }^{26} \mathrm{Im}$ plementation of existing and new guidelines is also problematic. This is not unique to genomic applications; only half of all adults in the United States receive recommended clinical preventive services, and just over half receive recommended care for acute and chronic conditions (e.g., Refs. 27-29). Therefore, research and evaluation of the dissemination and implementation of evidence recommendations relevant to genomic applications is essential. Many factors may influence clinical uptake of genomic applications such as reimbursement, physician knowledge/attitudes, patient interest/attitudes, and the presence of comparable applications. In the absence of such research, potential health benefits are unlikely to be fully realized throughout the US population.

We envision that recipients of current and planned translation research will become the initial nucleus of GAPPNet investigators, not only for conducting primary research in genomics translation but also for contributing expert knowledge synthesis and dissemination of research findings for use in health practice. A core group of such investigators already exists and is growing. Recognizing the existing evidence and dissemination dilemma in genomic medicine, the CDC recently launched a 
Table 2. Ongoing selected multigroup efforts in translating genomic discoveries into population health and health care in the United States

\begin{tabular}{|c|c|c|c|}
\hline Effort & Mission/purpose & Members & Activities \\
\hline IOM Genomics Roundtable ${ }^{43}$ & $\begin{array}{l}\text { "To advance the field of genomics and } \\
\text { improve the translation of research } \\
\text { findings to health care, education, } \\
\text { and policy" }\end{array}$ & Multiple & Workshops \\
\hline APHA Genomics Forum ${ }^{44}$ & $\begin{array}{l}\text { Members of the American Public } \\
\text { Health Association interested in } \\
\text { raising awareness and competencies } \\
\text { of genetics in public health }\end{array}$ & Public health & Committees policy statements \\
\hline $\mathrm{NCHPEG}^{45}$ & $\begin{array}{l}\text { "To promote health professional education } \\
\text { and access to information about } \\
\text { advances in human genetics to improve } \\
\text { the health care of the nation" }\end{array}$ & Multiple & Annual meetings, educational projects \\
\hline Genetic Alliance ${ }^{46}$ & "To transform health through genetics" & Disease support groups & $\begin{array}{l}\text { Annual meeting policy statements } \\
\text { special projects }\end{array}$ \\
\hline Personalized medicine coalition ${ }^{47}$ & $\begin{array}{l}\text { "To advance understanding and } \\
\text { adoption of personalized medicine } \\
\text { for the ultimate benefit of patients" }\end{array}$ & Multiple & Meetings, reports, advocacy \\
\hline $\begin{array}{l}\text { Network of State Health } \\
\text { Departments and Schools } \\
\text { of Public Health } 48\end{array}$ & $\begin{array}{l}\text { CDC funded four states projects to } \\
\text { integrate genomics into public health } \\
\text { programs and two schools with a } \\
\text { focus on chronic diseases }\end{array}$ & & Training surveillance education \\
\hline
\end{tabular}

translation research initiative in genomics to fund multiple groups to conduct translation research projects. ${ }^{30}$ This initiative focuses on the content identified through the genomic application horizon scans developed by the EGAPP Working Group and other tests and applications examined by USPSTF. These applications include the use of family history as a tool for disease prevention. Starting in 2009, several groups will be funded to conduct crucial research on candidate health applications. Some investigators might be groups already funded through NHGRIs Population genomics and ELSI programs. In addition, several National Institutes of Health $(\mathrm{NIH})$ initiatives have recently been announced. For example, the National Institute of Diabetes and Digestive and Kidney Diseases (NIDDK) of the NIH recently announced the availability of grants under the Secretary of Health and Human Services' "Genes, Environment, and Health Initiative," to conduct translation research to use results of new gene discoveries in clinical practice, psychosocial research as well as education and communication research. ${ }^{31}$ Also, the National Cancer Institute (NCI) at NIH currently is funding a number of investigators conducting translation research in genomics and personalized medicine and has several program announcements in this area (e.g., Refs. 32 and 33). The National Heart, Lung and Blood Institute (NHLBI) is conducting translation research on warfarin. In 2007, the FDA changed the labeling of warfarin to indicate that genetic information may affect warfarin dosing. Genetic information combined with clinical information may enhance patient treatment and outcomes, and personalize warfarin treatment for each individual. In a proof-of-concept study, NHBLI recently awarded a contract to conduct a large, multicenter, double-blind randomized trial of genotype-guided dosing of warfarin therapy, which is currently under way. ${ }^{34}$ Investigators from each of these new initiatives are excellent candidates for participation in GAPPNet.

\section{GAPPNet Domain 4: Programs to enhance practice, education, and surveillance}

In addition to building and disseminating a knowledge base and empowering translation research, GAPPNet will empower and promote translation programs focused on the implementation of validated genomic applications in practice and prevention, improving health care quality, and reducing costs. Health information technology has been identified as a key aspect of the genomics translation process, and there are a number of ongoing national activities designed to leverage health information technology to support genomics translation efforts. ${ }^{3}$ These activities will be conducted in population and clinical practice settings in the United States. In 2008, CDC released a Request for Applications to sponsor multiple groups including academia, health departments, and practice settings to perform such activities. ${ }^{35}$ Funding will be awarded in 2009. In addition, several groups are already conducting educational efforts and surveillance (some mentioned in Table 1). For example, the National Human Genome Research Institute of the NIH has awarded a contract for the development of a web-based curricular tool for interdisciplinary genomics education for nurses and physician assistant educators. Also, the Agency for Healthcare Research Quality (AHRQ) recently announced a new award to develop, implement and evaluate four computer-based decision-support tools that will help clinicians and patients, better use genetic tests to evaluate and treat breast cancer. The first tool will assess whether a woman with a family history of cancer should be tested for $B R C A 1$ and $B R C A 2$ gene mutations. The second tool will be used for women already diagnosed with breast cancer and will help determine which patients are appropriate candidates for gene expression profiling test. ${ }^{36}$

GAPPNet will interact with these groups to promote work related to the emerging list of candidate genomic applications in the knowledge database, especially the ones for which evi- 
dence-based guidelines have been developed. For example, with respect to the use of $B R C A 1$ testing in women at high risk because of family history, we currently have little evidence to date on utilization and impact on health outcomes in practice, especially since the publication of the USPSTF recommendation in 2005 (see examples of earlier studies ${ }^{37-40}$ ). Such programs are crucial in documenting attitudes, awareness and knowledge of consumers and providers, tracking integration and impact of genomic applications in practice as well as documenting and addressing issues of health disparities. The EGAPP Stakeholder Group and the initial cohort of CDC- and NIHfunded investigators will become active collaborators in GAPPNet and a cornerstone for its further development.

Examples of genomics translation programs activities are shown in Table 1. One illustration is the public health surveillance of consumer and provider awareness and use of direct to consumer tests. For the past few years, the CDC and several health departments have conducted a number of state and national surveys to assess knowledge, attitudes, and practices of such tests (e.g., BRCA $1^{41}$ and nutrigenomic tests ${ }^{42}$ ). Conducting translation research and programs will contribute valuable information to the knowledge base of "what does and what does not work" to improve health outcomes in the real world. Such an experience will stimulate additional research to close the knowledge gaps in genomic applications in practice and prevention.

\section{CONCLUDING REMARKS}

Despite the rapid evolution and success of human genome discoveries, the pace of implementation of genome-based applications in health care and population health has been slow. Major gaps exist in our understanding of how new knowledge of human genetic variation can be most effectively harnessed to improve health and prevent disease. We believe that GAPPNet will help to catalyze productive interactions between existing and emerging translational research efforts, thereby speeding knowledge base expansion and driving the dissemination of information for disease treatment and prevention. To further develop GAPPNet, CDC, and NIH will sponsor a number of stakeholder meetings to discuss goals, synergize activities, and develop specific action plans. In the meantime, readers of this article interested in the mission and vision of GAPPNet can find more information on the CDC public health genomics website available at: http://www.cdc.gov/genomics.

\section{ACKNOWLEDGMENTS}

The authors thank the following individuals for comments on the article: Elizabeth Gillanders, Dina Paltoo, Margaret Piper, Leah Sansbury, and Daniela Seminara.

\section{REFERENCES}

1. Manolio TA, Brook LD, Collins FS. A HapMap harvest of insights into the genetics of common disease. J Clin Invest 2008;118:1590-1605.

2. Feero WG, Guttmacher AE, Collins FS. The genome gets personal-almost. JAMA 2008;299:1351-1352.

3. Department of Health and Human Services. Personalized healthcare initiative. Available at: http://www.hhs.gov/myhealthcare/. Accessed July 21, 2008.

4. Secretary's Advisory Committee on Genetics, Health and Society. US system of oversight of genetic testing. Available at: http://www4.od.nih.gov/oba/ SACGHS/reports/SACGHS_oversight_report.pdf. Accessed July 21, 2008.

5. Khoury MJ, Bradley L, Berg A, et al. The evidence dilemma in genomic medicine: the need for a roadmap for translating genomic discoveries into clinical practice. Health Aff 2008;27:1600-1611.

6. Hudson KL, Holohan MK, Collins FS. Keeping pace with the times-the Genetic Information Nondiscrimination Act of 2008. N Engl J Med 2008; $358: 2661-2663$
7. Evaluation of Genomic Applications in Practice and Prevention (EGAPP). Available at: http://www.egappreviews.org/. Accessed January 1, 2009

8. Westfall JM, Mold J, Fagman L. Practice-based research - "blue highways" on the NIH road map. JAMA 2007;297:403-406.

9. Sung NS, Crowley WF, Genel M, et al. Central challenges facing the national clinical research enterprise. JAMA 2003;289:1278-1287.

10. Genome-based research and population health International Network (GRaPHInt). Available at: http://www.graphint.org. Accessed July 21, 2008.

11. Health Technology Assessment International. Available at: http://www. htai.org/. Accessed August 5, 2008.

12. Agency for Healthcare Research and Quality. Effective healthcare program. Available at: http://effectivehealthcare.ahrq.gov/aboutUs.cfm?abouttype=program. Accessed April 15, 2009.

13. Centers for Medicare and Medicaid Services. Coverage with evidence development. Available at: http://www.cms.hhs.gov/CoverageGenInfo/03_CED. asp. Accessed October 10, 2008.

14. Food and Drug Administration. Sentinel initiative. Available at: http://www. fda.gov/oc/initiatives/advance/sentinel/. Accessed October 10, 2008.

15. Health Resources and Services Administration. Maternal and Child Health Bureau. Available at: http://mchb.hrsa.gov/programs/. Accessed April 15, 2009.

16. National Institutes of Health. Clinical and Translational Sciences Awards. Available at: http://www.ncrr.nih.gov/clinical_research_resources/clinical_and_ translational_science_awards/. Accessed October 10, 2008.

17. Institute of Medicine Roundtable on Evidence-based Medicine. Available at: http://www.iom.edu/CMS/28312/RT-EBM.aspx. Accessed October 10, 2008.

18. Foundation for the National Institute of Health. Observational Medical Outcomes Partrnership. Available at: http://www.fnih.org/index.php?option= com_content\&task $=$ view\&id $=508 \&$ Itemid $=643$. Accessed October 10, 2008

19. Khoury MJ, Gwinn M, Yoon PW, Dowling N, Moore CA, Bradley C. "The continuum of translation research in genomic medicine" how can we accelerate the appropriate integration of human genome discoveries into health care and disease prevention? Genet Med 2007;9:665-674

20. Gene Tests. http://www.genetests.org. Accessed August 5, 2008.

21. Agency for Healthcare Quality Research. US Task Force on Preventive Services. Available at: http://www.ahrq.gov/clinic/USpstfix.htm. Accessed August 5, 2008.

22. Teutsch SM, Bradley LA, Palomaki G, et al. The Evaluation of Genomic Applications in Practice and Prevention (EGAPP) initiative: methods of the EGAPP working group. Genet Med 2009;11:3-14.

23. EGAPP. Recommendations from the EGAPP working group: can UGTIAI genotyping reduce morbidity and mortality in patients with metastatic colorectal cancer treated with irinotecan? Genet Med 2009;11:15-20.

24. EGAPP. Recommendations from the EGAPP working group: genetic testing strategies in newly diagnosed individuals with colorectal cancer aimed at reducing morbidity and mortality from Lynch syndrome in relatives. Genet Med 2009:11:35-41.

25. EGAPP. Recommendations from the EGAPP working group: can tumor gene expression profiling improve outcomes in patients with breast cancer? Genet Med 2009;11:66-73.

26. Nelson HD, Huffman LH, Fu R, Harris EL; U.S. Preventive Services Task Force. Genetic risk assessment and BRCA mutation testing for breast and ovarian cancer susceptibility: systematic evidence review for the U.S. Preventive Services Task Force. Ann Intern Med 2005;143:362-379.

27. Reid PP, Compton WD, Grossman JH, Fanjiang G, editors. Building a better delivery system. Washington, DC: National Academy of Sciences, National Academies Press, 2005

28. Schuster MA, McGlynn EA, Brook RH. How good is the quality of health care in the United States. Milbank $Q$ 2005;83:843-895.

29. McGlynn EA, Asch SM, Adams J, et al. The quality of health care delivered to adults in the United States. N Engl J Med 2003;348:2635-2645.

30. Centers for Disease Control and Prevention. Genomic applications in practice and prevention translation research. Funding announcement. Available at: http://www.cdc.gov/genomics/activities/fund2007_11_29.htm. Accessed August $5,2008$.

31. National Institutes of Health. Funding announcement. translation of common disease genetics into clinical applications. Available at: http://grants.nih.gov/ grants/guide/rfa-files/RFA-DK-08-004.html. Accessed August 5, 2008.

32. National Cancer Institute. Program announcements on development, application, and evaluation of prediction models for cancer risk and prognosis. Available at: http://grants.nih.gov/grants/guide/pa-files/PA-07-021.html. Accessed April 15, 2009

33. National Cancer Institute. Program announcement: understanding the effects of emerging cellular, molecular, and genomic technologies on cancer health care delivery. Available at: http:/grants.nih.gov/grants/guide/pa-files/PA-07260.html. Accessed August 5, 2008.

34. University of Pennsylvania. National Heart, Lung and Blood Institute. Genotyped guided dosing of warfarin clinical trial. Available at: http:// rt5.cceb.med.upenn.edu/warfdcc/WARF-1.html. Accessed September 22, 2008

35. Centers for Disease Control and Prevention. Genomic applications in practice 
and prevention translation programs in education, surveillance, policy. Funding announcement. Available at: http://www.cdc.gov/od/pgo/funding/GD08801.htm. Accessed August 5, 2008.

36. Agency for Healthcare Research Quality. Computer-based clinical decision support (CDS) tools for gene-based tests used in breast cancer. Available at: http://effectivehealthcare.ahrq.gov/healthInfo.cfm?infotype $=$ nr\&ProcessID $=68$. Accessed September 29, 2008.

37. Armstrong K, Micco E, Carney A, Stopfer J, Putt M. Racial differences in the use of BRCA1/2 testing among women with a family history of breast or ovarian cancer. JAMA 2005;293:1729-1736.

38. Halbert CH, Kessler L, Stopfer JE, Domchek S, Wileyto EP. Low rates of acceptance of BRCA1 and BRCA2 test results among African American women at increased risk for hereditary breast-ovarian cancer. Genet Med 2006;8:576-582.

39. Botkin JR, Smith KR, Croyle RT et al. Genetic testing for a BRCA1 mutation: prophylactic surgery and screening behavior in women 2 years post testing. Am J Med Genet A 2003;118:201-209.

40. Lerman C, Hughes C, Croyle RT et al. Prophylactic surgery and surveillance practices one year following BRCA1/2 genetic testing. Prev Med 2000;31: $75-83$

41. Myers M, Chang MH, Jorgensen C, et al. Genetic testing for susceptibility to breast and ovarian cancer: evaluating the impact of direct to consumer marketing campaign on physicians' knowledge and practices. Genet Med 2006;8:361-370.

42. Goddard KAB, Moore C, Ottman D, Szegda KL, Bradley L, Khoury MJ. Awareness and use of direct to consumer nutrigenomic tests: United States 2006. Genet Med 2007;9:510-517.

43. Institute of Medicine. Roundtable on translating genome-based research for health. Available at: http://www.iom.edu/CMS/3740/44443.aspx. Accessed August 5, 2008

44. American Public Health Association. Genomics Forum. Available at: http:/ aphagenomicsforum.org/index.php?note=AboutUs. Accessed August 5, 2008.

45. National Coalition for Health Professional Education in Genetics. Available at: http://www.nchpeg.org/. Accessed August 5, 2008.

46. Genetic Alliance. Available at: http://www.geneticalliance.org/. Accessed August 5, 2008

47. Personalized Medicine Coalition. Available at: http://www.personalizedmedicinecoalition.org/. Accessed August 5, 2008.

48. Centers for Disease Control and Prevention. National Office of Public Health Genomics. Genomics in practice. Available at: http://www.cdc.gov/genomics/ phpractice.htm. Accessed August 5, 2008. 